\title{
Activation of pancreatic stellate cells involves an EMT-like process
}

\author{
LEI TIAN ${ }^{1,3^{*}}$, ZI-PENG LU ${ }^{1-3^{*}}$, BAO-BAO CAI ${ }^{1-3}$, LIANG-TAO ZHAO $^{1,3}$, DONG QIAN $^{1,3}$, \\ QING-CHENG XU ${ }^{1,3}$, PENG-FEI WU ${ }^{1-3}$, YI ZHU ${ }^{1-3}$, JING-JING ZHANG ${ }^{1-3}$, \\ QING DU ${ }^{1-3}$, YI MIAO ${ }^{1-3}$ and KUI-RONG JIANG ${ }^{1-3}$ \\ ${ }^{1}$ Pancreas Institute of Nanjing Medical University; ${ }^{2}$ Pancreas Center, ${ }^{3}$ Laboratory for Department of General Surgery, \\ The First Affiliated Hospital of Nanjing Medical University, Nanjing 210029, P.R. China
}

Received August 10,2015; Accepted September 26, 2015

DOI: 10.3892/ijo.2015.3282

\begin{abstract}
Pancreatic adenocarcinoma (PDAC) and chronic pancreatitis (CP) are characterized by a desmoplastic reaction involving activated pancreatic stellate cells (PSCs). However, the mechanisms of PSC activation remain poorly understood. We examined whether the epithelial-mesenchymal transition (EMT) process might play a role in PSC activation. PSCs were isolated from a rat pancreas and characterized using immunofluorescence and immunocytochemistry. We evaluated changes in cell motility and in the expression levels of a panel of EMT-related genes during the PSC activation process. Activation of PSCs occurred after $48 \mathrm{~h}$ of in vitro culture, as indicated by a morphological change to a myofibroblastic shape and a decrease in the number of cytoplasmic lipid droplets. After activation, PSCs showed enhanced cell migration ability compared to quiescent cells. In addition, the expression of epithelial markers (E-cadherin, BMP7 and desmoplakin) decreased, while expression of mesenchymal markers (N-cadherin, vimentin, fibronectin1, collagen $1 \alpha 1$ and S100A4) increased in activated PSCs. EMT-related transcription factors (Snail and Slug) were also upregulated after PSC activation. The concurrent increase in cell migration ability and alterations in EMT-related gene expression suggests that the activation of PSCs involves an EMT-like process. The knowledge that PSC activation involves an EMT-like process may help to identify potential new therapeutic targets to alleviate pancreatic fibrosis in diseases like $\mathrm{CP}$ and PDAC.
\end{abstract}

Correspondence to: Professor Yi Miao or Professor Kuirong Jiang, Pancreas Center, The First Affiliated Hospital of Nanjing Medical University, 300 Guangzhou Road, Nanjing 210029, P.R. China

E-mail: miaoyi@njmu.edu.cn

E-mail: jiangkuirong@njmu.edu.cn

${ }^{*}$ Contributed equally

Key words: pancreatic stellate cells, activation, epithelial-mesenchymal transition, pancreatic adenocarcinoma, chronic pancreatitis

\section{Introduction}

Pancreatic cancer is the fourth leading cause of cancer-related deaths in men and women in the US (1). The most common type of pancreatic cancer is pancreatic ductal adenocarcinoma (PDAC). PDAC is one of the most lethal cancers with a low 5-year survival rate and limited therapeutic options. Although the etiology of PDAC is still unclear, chronic pancreatitis (CP) has been identified as one of the potential precursors of PDAC $(2,3)$. A relationship between CP and PDAC has been elucidated in rodent disease models (4). In both CP and PDAC, a desmoplastic reaction occurs in the pancreas stroma, characterized by a collagen-rich extracellular matrix (ECM) and activated associated inflammatory cells (5-8). One of the important reasons that chemotherapy does not work well in PDAC patients is because drugs are poorly delivered to the tumor due to this dense stroma abundant with collagens, glycoproteins and low-density vasculature $(9,10)$.

It has been demonstrated that activated pancreatic stellate cells (PSCs) play a central role in the desmoplastic reaction in the pancreas (11-13). PSCs were first described by Watari et al in 1982 (14), and were successfully isolated by Apte et al (15) and Bachem et al in 1998 (16). Under normal physiological conditions, PSCs are mainly located in periacinar and interlobular areas of the pancreas and maintain a quiescent phenotype characterized by abundant vitamin A-containing lipid droplets in the cytoplasm (15). These quiescent PSCs keep the balance between ECM synthesis and degradation. However, in diseases such as CP and PDAC, PSCs transform or transdifferentiate into an activated phenotype, which are devoted to stroma remodeling (17). During this transdifferentiation process, PSCs change their morphology and function as follows: i) they change into a myofibroblastic shape and lose their vitamin-A containing lipid droplets; ii) they acquire migration and ECM modulation capacity; and iii) they upregulate expression of $\alpha$-SMA, vimentin, desmin and GFAP. While scientists have previously tried to control or reverse this process of PSC activation, the mechanisms involved in the activation process are not fully clarified.

Activated PSCs not only act as positive regulators of cell proliferation and migration, but inhibit tumor cell apoptosis. In turn, cancer cells can facilitate the activation, proliferation, migration and ECM production of PSCs. Such features 
prompt local tumor progression as well as regional and distant metastasis $(18,19)$. Therefore, a better understanding of the mechanisms of PSC transdifferentiation is crucial for research on the pathogenesis and clinical treatment of $\mathrm{CP}$ and PDAC.

PSC activation shares many common morphological and functional changes with the epithelial-mesenchymal transition (EMT) process. EMT is the basis for wound healing in response to chronic injury induced by toxic, viral, metabolic, or immunological factors $(20,21)$. The EMT process turns an epithelial cell into a mesenchymal cell and has been widely studied in the field of embryogenesis, tissue fibrosis and cancer metastasis (22-24). Typically, EMT is characterized by the expression of specific cell-surface proteins and cytoskeletal proteins, as well as changes in ECM proteins production, followed by activation of transcription factors. For example, EMT is characterized by upregulated expression of vimentin and $\mathrm{N}$-cadherin, and downregulated expression of E-cadherin, accompanied by enhanced expression of transcription factors, such as Snail, Slug, and Twist $(25,26)$. The EMT process is divided into three different types (1-3). Type 2 EMT is characterized by conversion from endothelial or epithelial cells into fibroblasts, which are responsible for tissue inflammation and fibrosis, such as that seen in PDAC $(27,28)$. Morphological changes, with redistribution of stress fibers and increased migratory capacity, are other main features of the EMT process.

Due to these similarities between EMT and PSC activation, we hypothesized that activation of PSCs may involve an EMT-like process. Indeed, it has previously been reported that an EMT-like process is involved in transdifferentiation of quiescent hepatic stellate cells (HSCs), a homology of PSCs in the liver (29). We tested our hypothesis by examining the functional alterations and expression of EMT-related genes during the activation process of PSCs in vitro. By studying this process of PSC activation, we aim to uncover novel therapeutic targets for treatment of CP and PDAC.

\section{Materials and methods}

Harvesting rat pancreas. All animal studies were reviewed and approved by the Ethics Committee of Nanjing Medical University in accordance with the established standards of the humane handling of research animals. Male Sprague-Dawley rats (150-300 g) were obtained from Vital River Laboratories (Beijing, China). Rats were kept in standard laboratory conditions with light-dark cycles of 12-12 $\mathrm{h}$ and free access to chow and water. Rats were sacrificed by decapitation, before undergoing laparotomy. Rat pancreas was dissected and placed into cold $0.9 \% \mathrm{NaCl}$ solution.

Isolation of quiescent PSCs. Quiescent PSCs were isolated from rat pancreas with enzyme digestion and Nycodenz gradient centrifugation as described previously (15). Briefly, after connective tissue and blood vessels were removed, pancreatic tissue was slowly infused with $10 \mathrm{ml}$ enzyme solution (mixture of collagenase P, protease and DNase) until all lobules were swollen and well infused. The pancreas tissue was pre-incubated, finely minced and incubated again, in order to obtain a cell suspension. After washing, PSCs were obtained by ladder centrifugation with Nycodenz solution. Cells were cultured in DMEM/F12 (Wisent, Canada) supplemented with $10 \%$ fetal bovine serum (FBS) (Wisent, Canada), $4 \mathrm{mM}$ L-glutamine (Sigma-Aldrich, St. Louis, MO, USA) and $1 \%$ penicillin-streptomycin (Thermo Scientific Hyclone, Waltham, MA, USA) at $37^{\circ} \mathrm{C}$ with $5 \% \mathrm{CO}_{2}$. Cells were subcultured at $90 \%$ confluence in the following passages.

Characterization and identification of isolated cells. Morphological appearances of isolated cells were assessed under a contrast-phase microscope with blue-green autofluorescence at $320 \mathrm{~nm}$ from lipid droplets within the cytoplasm. PSCs were evaluated by immunocytochemistry/immunofluorescence (ICC/IF) and immunocytochemistry (ICC) using antibodies specific for PSC markers, $\alpha$-SMA, desmin, vimentin, and GFAP. Antibodies for epithelium and macrophage markers (pan-CK, CK19, CD68) were used as negative controls to rule out possible cell contamination. Cells were seeded and stained in a 24-well plate (Corning Inc., NY, USA). ICC/IF and ICC were performed with standard procedures. The primary antibodies and their working dilutions were as follows: rabbit anti- $\alpha$-SMA 1:100 (Abcam, Cambridge, MA, USA); rabbit anti-desmin 1:200 (Abcam); rabbit anti-vimentin 1:100 (Cell Signaling Technology, Beverly, MA, USA); mouse anti-GFAP 1:500 (Sigma-Aldrich); rabbit anti-CK19 1:300 (Abcam); mouse anti-CD68 1:100 (Abcam); and mouse antipan-CK 1:100 (Thermo Fisher Scientific, USA). DAPI and hematoxylin were used for nuclear counterstaining in ICC/IF and ICC, respectively. Cells were observed under a fluorescence microscope (Nikon Ti, Japan).

Cell migration assay. Cell migration assays were carried out in 24-well transwell chambers with polycarbonate filters (pore size, $8 \mu \mathrm{m}$; Merck Millipore, Billerica, MA, USA). A total of $2 \times 10^{4}$ cultured cells, at $40 \mathrm{~h}$ and on day 10 after isolation, were plated in serum-free medium in the upper chamber, and media containing 10\% FBS alone was added to the lower chamber. After $40 \mathrm{~h}$ of incubation, residual medium in the upper chamber was removed carefully with a pipette. Cells on the surface of the filter membrane were fixed and stained with crystal violet staining solution (Beyotime, Shanghai, China) according to the manufacturer's instructions. Penetrated cells on the lower surface of the membrane were examined and counted under the microscope, before being removed with a cotton swab. Non-penetrated cells were assessed via the same method. Migration rates were calculated with the following formula: number of penetrated cells divided by the total number of both penetrated and non-penetrated cells.

Live cell motility assay. For cell motility studies of PSC, a live cell imaging system was used. There are many essential elements for this system. Firstly, a camera was mounted on the pressurized chamber on the stage of a Nikon TE 2000-E inverted optical microscope. Secondly, the microscope employed a number of computer controlled motorized systems in order to rapidly reconfigure the microscope during automated image acquisition. Finally, a small cell incubator on the microscope was used to control the cell environment and maintain our samples in a humidified and $\mathrm{CO}_{2}$ enriched atmosphere. The system was kept in a darkened room with ambient 

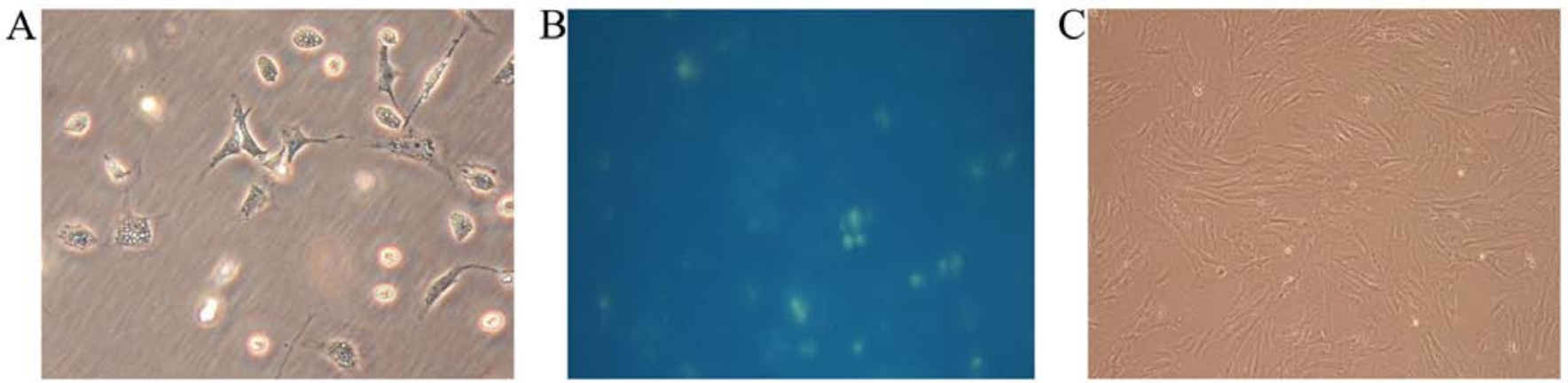

Figure 1. Isolation of rat pancreatic stellate cells (PSCs) in vitro. (A) Light microscopy of quiescent PSCs after $40 \mathrm{~h}$ in culture, magnification, $\mathrm{x} 200 . \mathrm{Cells}$ were round or polygon in appearance and showed abundant lipid droplets in the cytoplasm. (B) Transient blue-green autofluorescence at 320 nm could be examined due to the presence of vitamin A in the cytoplasm after $24 \mathrm{~h}$ in culture, magnification, $\mathrm{x} 200$. (C) Cell culture 10 days after isolation showing that PSCs developed a fibroblast-like morphology and lost most of their lipid droplets, magnification, x100.

temperature maintained close to $25^{\circ} \mathrm{C}$. The entire process was controlled by NIH-Elements AR software, and the following image processing was performed using Image-Pro Plus 6.0. Cells were cultured in 6-well plate and single cell movements was tracked with automatic record of a series of continuous images at an interval of 2 min under 100 magnified vision over $72 \mathrm{~h}$. Cell motility capacity was assessed with the length of cell movement track over a fixed time.

Total RNA isolation and quantitative RT-PCR. Total RNA was extracted from quiescent and activated PSCs cultured in 6-cm Petri dishes with Qiagen RNeasy Micro kit (Qiagen, Mannheim, Germany) according to the manufacturer's instructions. Agarose gel electrophoresis was used for quality control of the isolated RNA. Reverse transcription was conducted using PrimeScript RT Master Mix (Takara, Dalian, China), while quantitative reverse transcription polymerase chain reaction (qRT-PCR) was performed using FastStart Universal SYBR Green Master (ROX) (Roche, Germany) on a StepOne Plus Real-Time-PCR System (Applied Biosystems, Waltham, MA, USA) according to the manufacturer's instructions. The PCR program consisted of an initial enzyme activation step at $95^{\circ} \mathrm{C}$ for $10 \mathrm{~min}$, followed by 45 cycles of amplification with $95^{\circ} \mathrm{C}$ for $15 \mathrm{sec}$, followed by incubation at an appropriate temperature for $1 \mathrm{~min}$. Finally, a melting curve profile was set at $95^{\circ} \mathrm{C}(15 \mathrm{sec}), 60^{\circ} \mathrm{C}(1 \mathrm{~min})$, and $95^{\circ} \mathrm{C}(15 \mathrm{sec}) . \beta$-actin was used as an endogenous control to which each gene of interest was normalized. Relative quantification was calculated by the $\Delta \Delta \mathrm{CT}$ method and normalized based on the designated control. Primer sequences used in qRT-PCR are shown in Table I.

Western blotting. Total proteins were prepared with a protein extraction kit (Beyotime) and quantified by the bicinchoninic acid assay (BCA) method (Beyotime) according to the manufacturer's instructions. Polyvinylidene fluoride (PVDF) membranes (Millipore) were blocked in 5\% non-fat dried milk in phosphate-buffered saline with Tween-20 (PBST) and incubated overnight at $4^{\circ} \mathrm{C}$ with appropriate primary antibodies [rabbit anti- $\alpha$-SMA, dilution 1:200 (Abcam); rabbit anti-vimentin, dilution 1:1,000 (Cell Signaling Technology); rabbit anti-S100A4, dilution 1:250 (Abcam); rabbit anti-Ncadherin, dilution 1:200 (Santa Cruz Biotechnology, Dallas,
TX, USA); mouse anti-E-cadherin, dilution 1:1,000 (Abcam); rabbit anti-BMP7, dilution 1:1,000 (Abcam); rabbit anti-Slug, dilution 1:700 (Abcam); and rabbit anti-Snail, dilution 1:1,000 (Cell Signaling Technology)]. Membranes were then washed and incubated for $2 \mathrm{~h}$ at room temperature with secondary horseradish peroxidase-conjugated anti-mouse IgG or antirabbit IgG (Cell Signaling Technology). All protein expression levels were normalized to $\beta$-actin (Abcam).

Statistical analysis. Statistical analysis was performed using SPSS, version 13.0 (SPSS, Inc., Chicago, IL, USA). All experiments were repeated in triplicate, and the most representative data are shown in this report. Numeric data were expressed as mean \pm standard deviation (SD). Statistical significance between two groups was determined by the Student's t-test, with P-values $<0.05$ considered statistically significant.

\section{Results}

Isolation and identification of rat quiescent and activated PSCs. The yield of rat quiescent PSCs was $\sim 4$ to 7 million cells per gram of pancreatic tissue. Freshly isolated PSCs remained quiescent within the first $48 \mathrm{~h}$ of primary culture as indicated by their typical round or polygon shape and autofluorescence from lipid droplets in their cytoplasm (15). After $48 \mathrm{~h}$, they began to transform to an 'activated' form, acquiring fibroblastic characteristics and losing their lipid droplets. Apte et al previously demonstrated that freshly isolated PSCs cultured on plastic were gradually activated after $48 \mathrm{~h}$ and fully activated after 7 days in vitro (15). For this reason, we chose cells cultured for $40 \mathrm{~h}$ after isolation as representative of the quiescent state and cells cultured for 10 days as representing the fully activated state (Fig. 1). Primarily cultured cells were positive for PSC marker ( $\alpha$-SMA, vimentin, GFAP and desmin) staining in ICC and ICC/IF (Fig. 2). To exclude possible contamination with macrophages and epithelial cells, the purity of PSCs were confirmed with negative immunostaining with CK19, CD68, and pan-CK (Fig. 3).

Cell migration ability is promoted after PSC activation. PSC migration ability was assessed using transwell chambers with 40-h incubation time. More cells migrated to the lower surface 
Table I. Sequences of primers used in qRT-PCR for EMT related genes.

\begin{tabular}{|c|c|c|c|c|}
\hline Gene & Accession no. & Primer sequence $\left(5^{\prime}-3^{\prime}\right)$ & Amplicon (bp) & $\mathrm{Ta}$ \\
\hline rat S100A4 & NM_012618.2 & $\begin{array}{l}\text { Forward: TCCACCTTCCACACATACTCAG } \\
\text { Reverse: TTCATTGTCCCTGTTGCTGT }\end{array}$ & 168 & 55 \\
\hline rat Vimentin & NM_031140.1 & $\begin{array}{l}\text { Forward: ATGTCCGCCAGCAGTATG } \\
\text { Reverse: CCTGTCTCCGGTATTCGTTT }\end{array}$ & 156 & 56 \\
\hline rat Collagen $1 \alpha 1$ & NM_053304.1 & $\begin{array}{l}\text { Forward: CTGCCCAGAAGAATATGTATCAC } \\
\text { Reverse: GAAGCAAAGTTTCCTCCAAG }\end{array}$ & 198 & 58 \\
\hline rat Fibronectin 1 & NM_019143.2 & $\begin{array}{l}\text { Forward: GCCCTTACAGTTCCAAGTTCC } \\
\text { Reverse: TTGTGCCTCCTCTGGTTGTG }\end{array}$ & 114 & 55 \\
\hline rat $\mathrm{N}$-cadherin & AB017695.1 & $\begin{array}{l}\text { Forward: CTGAATGGGATGCTGAGGT } \\
\text { Reverse: TTGAAAGGCCGTAAGTGGG }\end{array}$ & 193 & 55 \\
\hline rat E-cadherin & NM_031334.1 & $\begin{array}{l}\text { Forward: ACAGGCCAGAGTTTATCCAGG } \\
\text { Reverse: TGAGGATGGTGTAGGCGATG }\end{array}$ & 144 & 55 \\
\hline rat BMP7 & NM_001191856.1 & $\begin{array}{l}\text { Forward: AAAACAGCAGCAGTGACCAG } \\
\text { Reverse: TTCGTGTAGGAGTTCAGAGG }\end{array}$ & 157 & 54 \\
\hline rat Desmoplakin & ВC098071.1 & $\begin{array}{l}\text { Forward: GGTCTGGTAGGCATTGAGTT } \\
\text { Reverse: AGTTCCTTGTTCATCGCTTG }\end{array}$ & 119 & 52 \\
\hline rat Slug & U97061.1 & $\begin{array}{l}\text { Forward: GGAGCGTACAGCCCTATAACT } \\
\text { Reverse: CTAATGGGACTTTCTGAACCAC }\end{array}$ & 170 & 55 \\
\hline rat Snail1 & NM_053805.1 & $\begin{array}{l}\text { Forward: AGTTGTCTACCGACCTTGCG } \\
\text { Reverse: TGCAGCTCGCTATAGTTGGG }\end{array}$ & 128 & 52 \\
\hline rat HHIP & NM_001191817.1 & $\begin{array}{l}\text { Forward: AATGTGAGCCACCTTGTCGT } \\
\text { Reverse: TCACACTGAGGGCCGAGATA }\end{array}$ & 88 & 50 \\
\hline rat Gli1 & NM_001191910.1 & $\begin{array}{l}\text { Forward: GGACTTTCTGGTCTGCCCTT } \\
\text { Reverse: AGATGGAAAGAGCCCGCTTC }\end{array}$ & 157 & 54 \\
\hline rat Gli2 & NM_001107169.1 & $\begin{array}{l}\text { Forward: TAAGCGGAGCAAGGTCAAG } \\
\text { Reverse: GTGGCAGTTGGTCTCGTAGAT }\end{array}$ & 154 & 57 \\
\hline rat Shh & NM_017221.1 & $\begin{array}{l}\text { Forward: CATCCCTTGGGAATGGCAGT } \\
\text { Reverse: TGCTTATCTGGCAGTCGCTT }\end{array}$ & 98 & 52 \\
\hline rat ACTB & NM_031144.3 & $\begin{array}{l}\text { Forward: TGTGCTATGTTGCCTCAGACT } \\
\text { Reverse: CATTGCCGATAGTGATGACCT }\end{array}$ & 111 & 55 \\
\hline
\end{tabular}

Ta, annealing temperature.

of the membrane 10 days after isolation compared with those isolated after only $40 \mathrm{~h}$. In order to rule out possible differences in cell adherence and proliferation ability after activation, the cell migration rate was calculated. Increased cell migration rate was observed after PSC activation (Fig. 4).

Live cell motility rate is increased after PSC activation. Cell motility capacity was assessed by live cell imaging system. PSC motility capacity was increased after cell activation, as the activated PSCs moved a longer distance during a fixed culture time. The real-time images of randomly chosen monitored cells are showed in Fig. 5. At the same time, PSCs underwent characteristic morphological changes in vitro, including loss of lipid droplets, increased cell volume and transition to a myofibroblastic phenotype, which is in accordance with our previous findings $(15,16)$.

Changes in the expression of an EMT-related gene panel after PSCs activation. To determine whether PSC activation involve an EMT-like process, we analyzed expression of epithelial markers E-cadherin, bone morphogenetic protein 7 (BMP7), and desmoplakin, and the mesenchymal markers $\mathrm{N}$-cadherin, fibroblast-specific protein 1 (S100A4), vimentin, fibronectin 1 , and collagen $1 \alpha 1$, during the PSC activation 

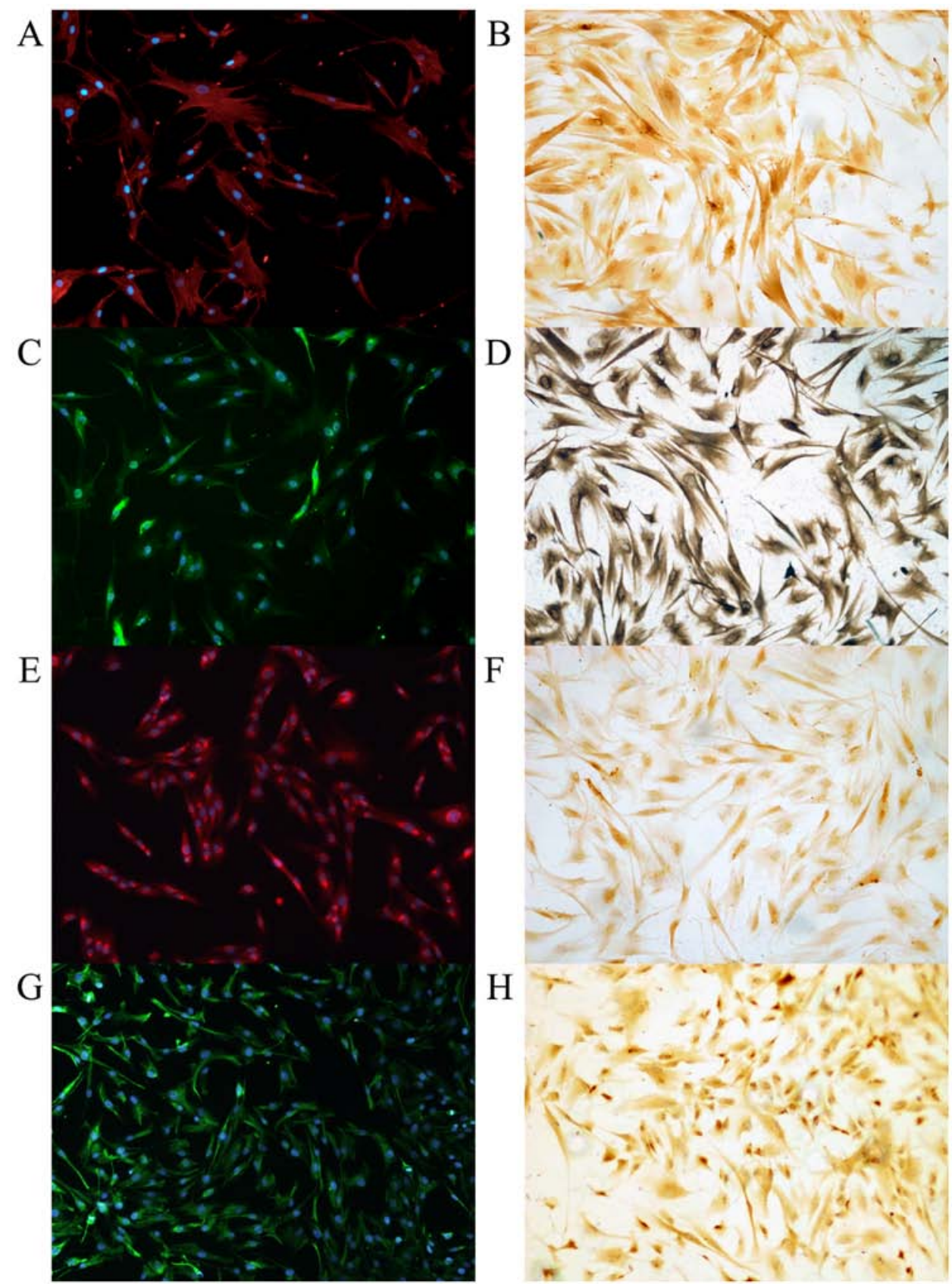

Figure 2. Expression of cellular markers in isolated pancreatic stellate cells (PSCs), magnification, x100. (A, C, E and G) Immunofluorescence staining for isolated PSCs. DAPI (blue) was used to counterstain the nuclei. (B, D, F and G) Immunocytochemistry staining for isolated PSCs. Hematoxylin (purple) was used to counterstain the nuclei. (A and B) $\alpha$-SMA [(A) red; (B) brown]. (C and D) Vimentin [(C) green; (D) brown]. (E and F) GFAP [(E) red; (F) brown]. $(\mathrm{G}$ and $\mathrm{H})$ Desmin $[(\mathrm{G})$ green; $(\mathrm{H})$ brown $]$.
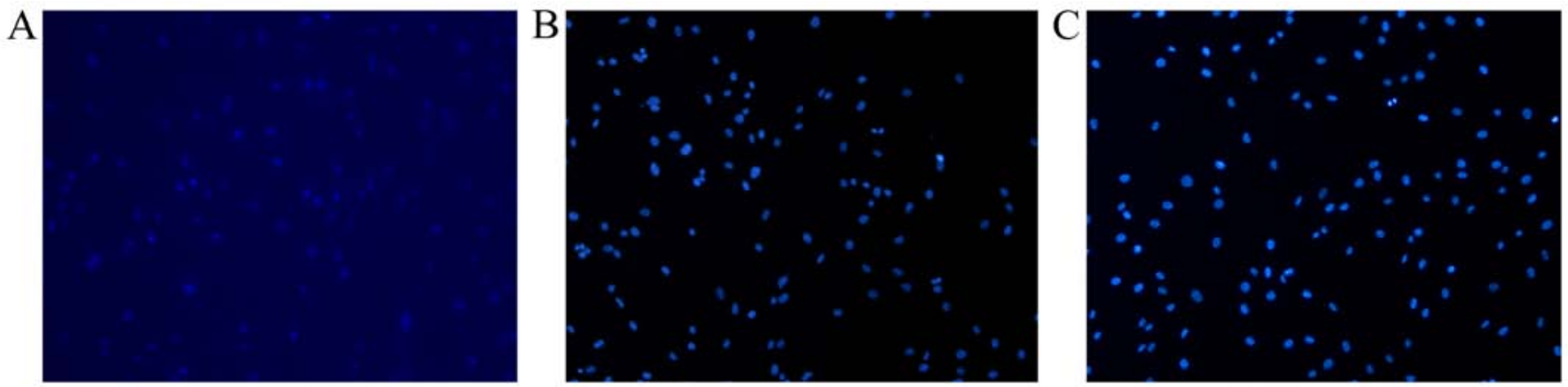

Figure 3. Immunofluorescence staining of macrophage and epithelium markers in isolated pancreatic stellate cells (PSCs), magnification, x100. DAPI (blue) was used to counterstain the nuclei. (A) CK19; (B) CD68; (C) pan-CK [if positively stained (A) was green, while (B) and (C) were red]. 

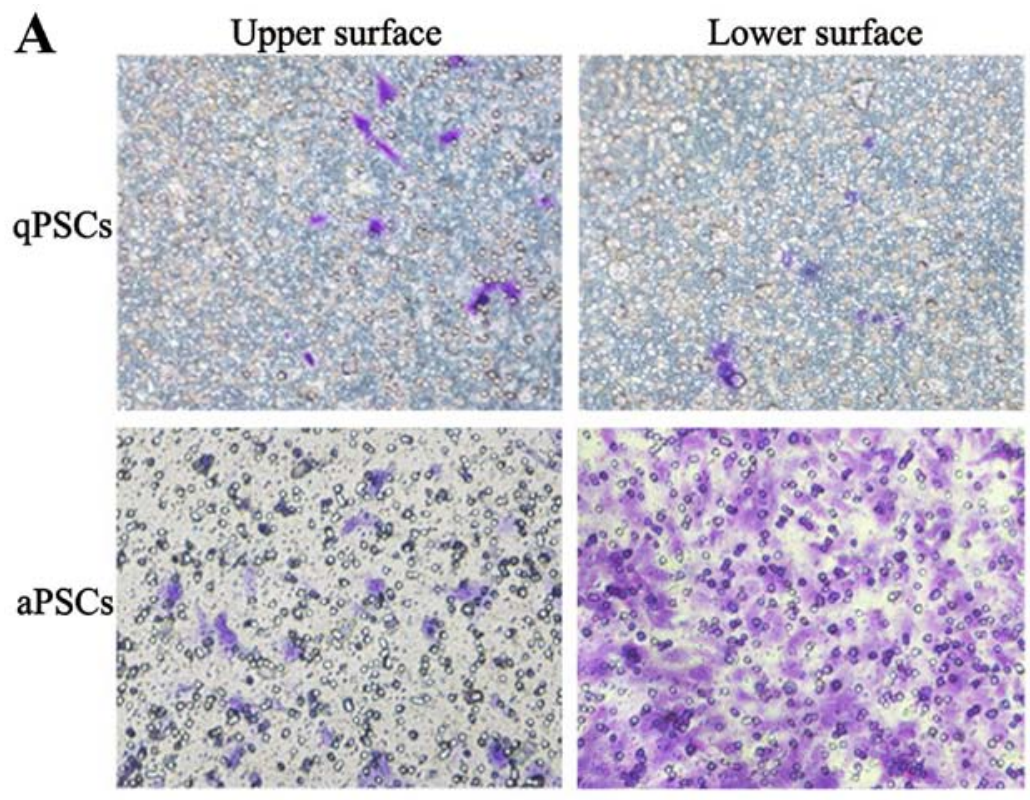

\section{B}
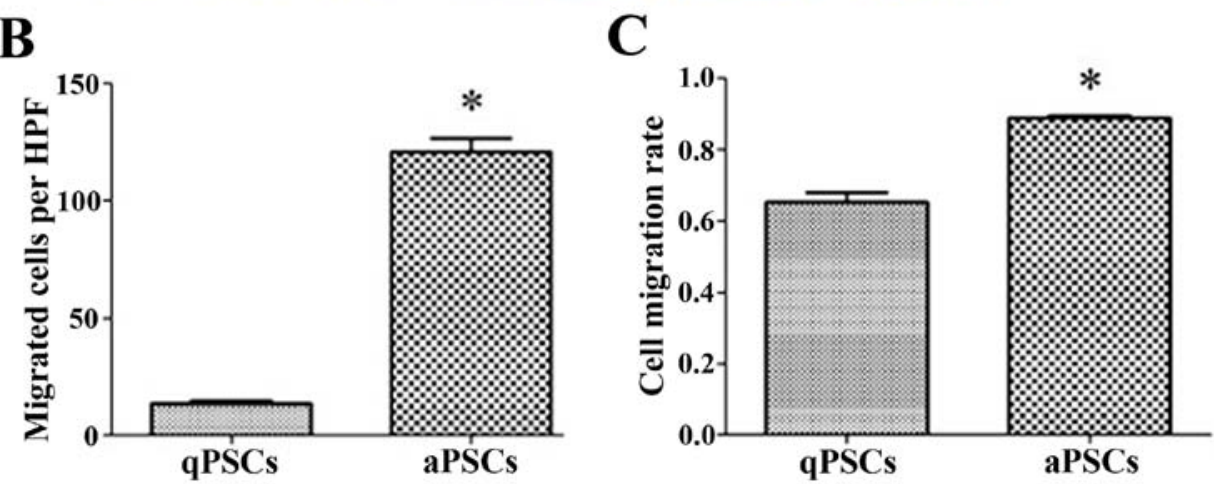

Figure 4. Activation of pancreatic stellate cells (PSCs) promotes their migration ability (qPSCs, quiescent PSCs, and aPSCs, activated PSCs). (A) Cells were fixed, staining and counted after $40 \mathrm{~h}$ of a transwell migration assay. Cells on the upper surface and lower surface of polycarbonate membrane represent nonpenetrated and penetrated cells, respectively. (B) Significantly more cells migrated after activation compared with their quiescent counterparts, as indicated by more cells in the lower surface after migration assay. Data are expressed as mean \pm SD from three biological replica, ${ }^{*} \mathrm{P}<0.05$. (C) An increased migration rate was observed in activated PSCs (10 days after isolation), compared with quiescent cells. Data are expressed as mean \pm SD from three biological replica, ${ }^{*} \mathrm{P}<0.05$.

process by qRT-PCR. E-cadherin, BMP7 and desmoplakin were significantly downregulated, while N-cadherin, S100A4, vimentin, fibronectin 1 and collagen $1 \alpha 1$ were upregulated in activated PSCs after 10 days of in vitro culture compared with quiescent PSCs (Fig. 6).

Since the Snail transcription factor family, such as Snail and Slug (also known as Snail1 and Snail2), is reported to play a crucial role during the EMT process, we used qRT-PCR to examine the expression of Snaill and Slug during PSC activation. Both Snail1 and Slug mRNA levels increased significantly in activated PSCs compared to quiescent PSCs. We also examined the expression of EMT-related genes at the protein level by immunoblotting. Western blot analysis confirmed that the decreased protein expression of epithelial markers, including E-cadherin and BMP-7, was accompanied by increased expression of mesenchymal markers (i.e., N-cadherin, vimentin, S100A4) after PSCs were activated (Fig. 7). Furthermore, the two EMT-related transcription factors, Snail and Slug, also showed increased expression at the protein level during PSC activation.

\section{Discussion}

Previous studies proved that PSCs stimulated the EMT process of cancer cells, and thereby elevated tumor migration and invasion (30). The present study provides novel evidence that PSC activation induced EMT-related gene expression in vitro. We also observed significant alterations in morphology and migration capacity when PSCs were fully activated. We found that PSC activation was accompanied by downregulation of E-cadherin and upregulation of $\mathrm{N}$-cadherin, vimentin, collagen $1 \alpha 1$ and fibronectin1 gene expression.

Besides the classical molecular regulators of EMT described above, we also examined expression of BMP7 and S100A4. Bone morphogenetic proteins such as BMP7 belong to a large family of cytokines, which regulate various biological processes including cell proliferation, apoptosis, differentiation and morphogenesis (31). A close relationship between BMP signaling and EMT during embryogenesis, fibrosis and cancer development has been elucidated by several studies (32-34). BMP7, in particular is recognized as an antagonist of 


\section{A: Quiescent PSCs}

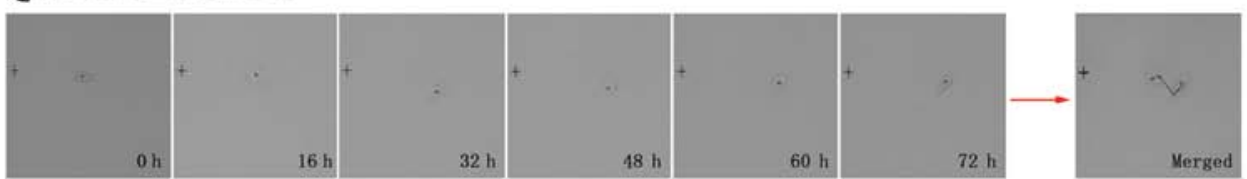

Zoom A

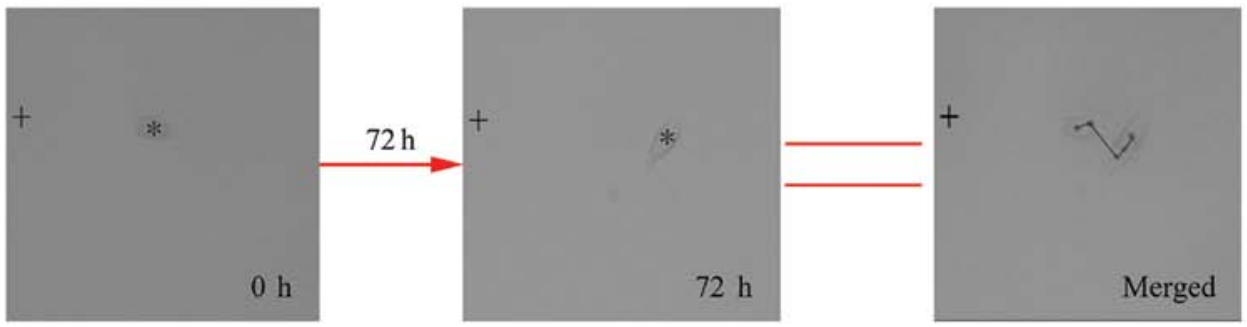

B: Activated PSCs

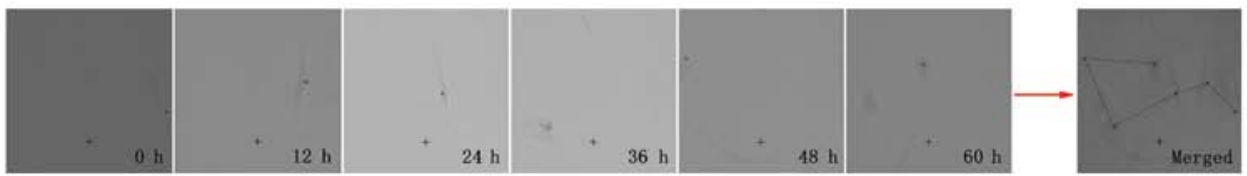

Zoom B
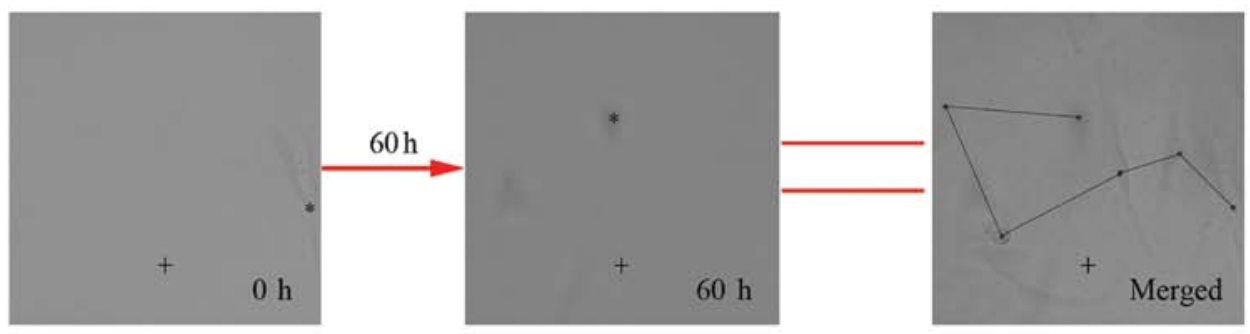

C

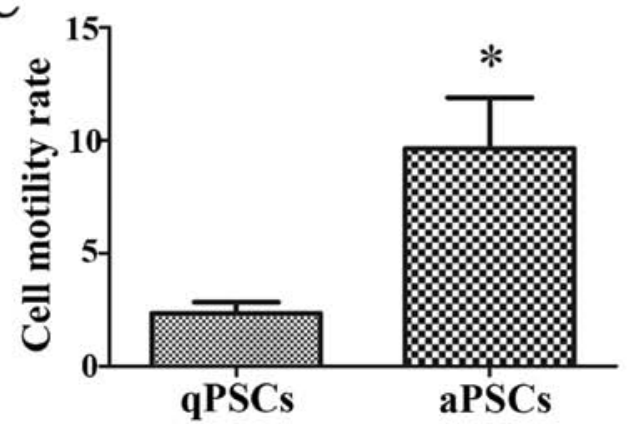

Figure 5. Changes in pancreatic stellate cell (PSC) motility capacity after cell activation as assessed by live cell imaging system. Asterisks indicate the chosen single cell under the microscope, and the cross indicates the fixed reference in the continuous imaging. (A) Continuous imaging of quiescent PSC cultured in live cell imaging system for $72 \mathrm{~h}$. The cell movement track is shown in the last image (merged). (B) Continuous imaging of activated PSC (10 days after isolation) cultured in live cell imaging system for $60 \mathrm{~h}$. The cell movement track is shown in the last image (merged). (C) Cell motility capacity assessed by the average length of the movement track of five randomly selected single cells over the culture time. Motility capacity was improved in activated PSCs compared to quiescent cells, which is indicated by an increased length of their movement tracks. Data are expressed as mean \pm SD from three biological replica, ${ }^{*} \mathrm{P}<0.05$.

EMT induced by TGF- $\beta(35,36)$. In this study, BMP7 expression was significantly decreased in activated PSCs.

S100A4 is a member of the calcium-binding S100 protein family, and has been associated with cell proliferation, cellular adhesion, reconstruction of the ECM, angiogenesis and cellular motility (37-39). Cells that express both $\alpha$-SMA and S100A4 are identified as fibroblasts (40) and S100A4 is expressed in fibroblasts during type 2 EMT (41). In our study, when PSCs maintained a fully activated state, S100A4 expression was notably increased.
Furthermore, the present study proves that the Snail and Slug transcription factors were related to the activation of PSCs. The vertebrate Snail genes mediating EMT are divided into two subtypes: Snaill and Snail2 (also known as Slug) $(42,43)$. Snail1 and Snail2 are known as repressors of E-cadherin and can upregulate mesenchymal markers such as vimentin $(44,45)$. Previous research showed that Snail expression was associated with tissue fibrosis (42). In addition, Scarpa et al reported that Snaill plays a pivotal role in HSC activation (46). Similarly, in this study, Snaill and Slug were upregulated in PSC activation. 
Epithelial markers:

BMP7

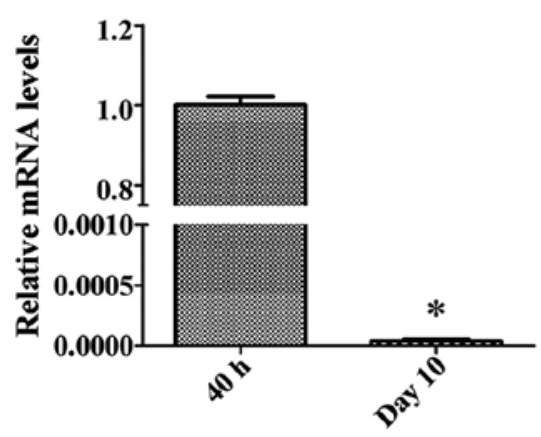

Mesenchymal markers:

\section{Collagen1 $\alpha 1$}

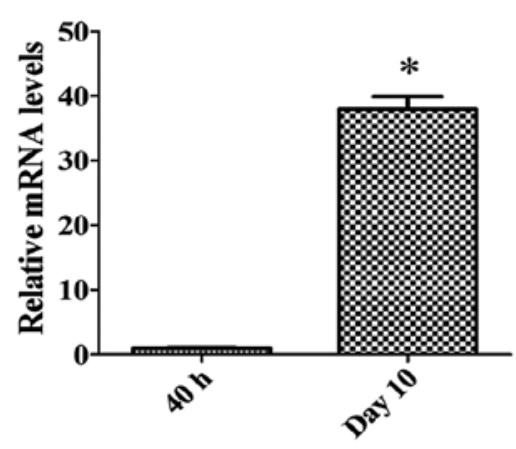

Vimentin

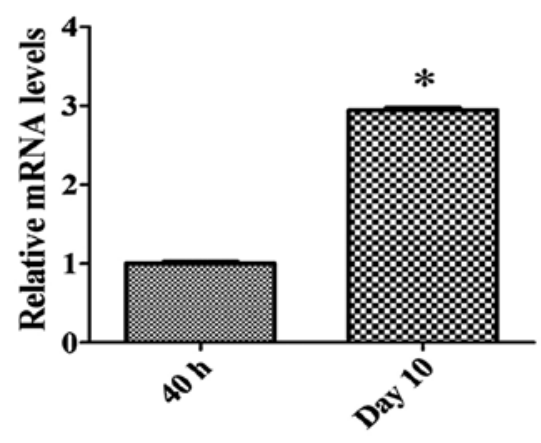

\section{Transcription factors:}

Snail1

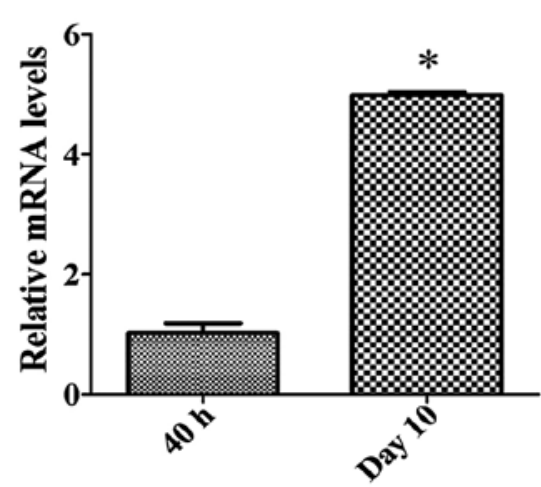

Desmoplakin

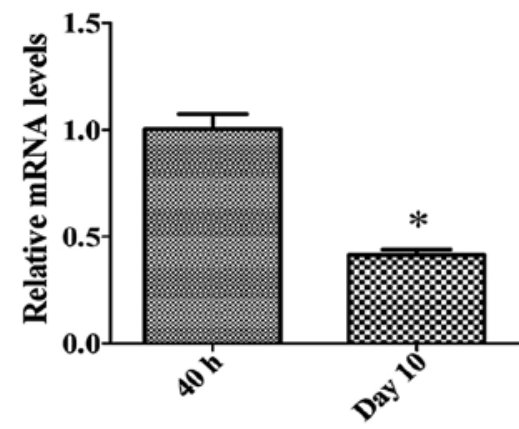

Fibronectin1

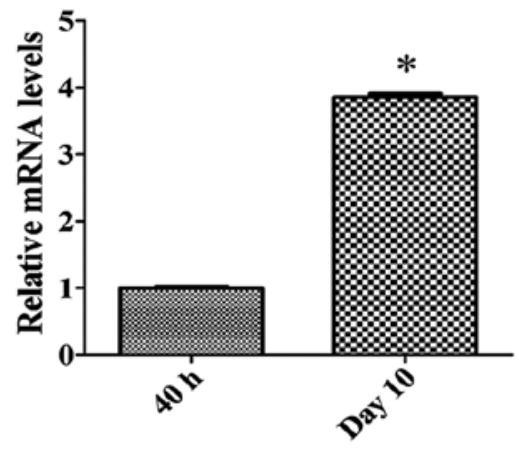

S100A4
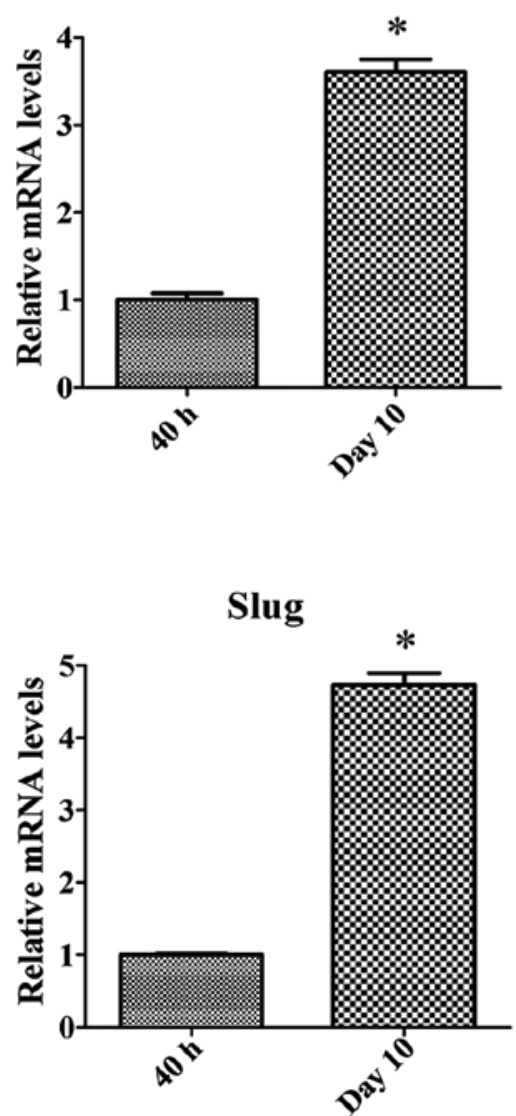

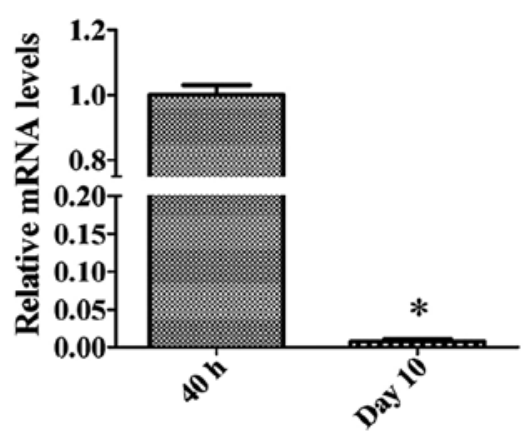

N-cadherin

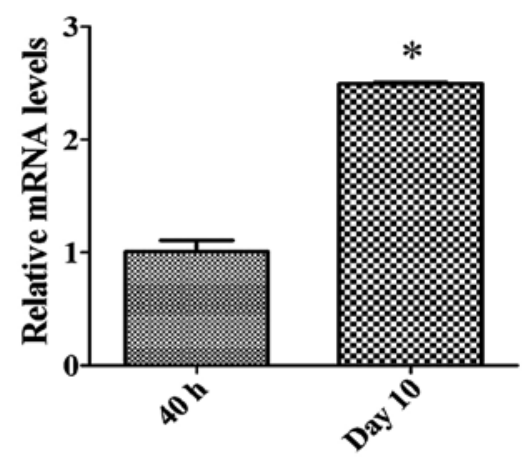

Figure 6. Quantitative real-time PCR indicating that pancreatic stellate cell (PSC) activation is accompanied by alterations in expression of a panel of EMTrelated genes. Compared to quiescent PSCs ( $40 \mathrm{~h}$ after isolation), there were significantly decreased expression of epithelial markers (E-cadherin, BMP7 and desmoplakin), together with increased expression of mesenchymal markers (collagen1 $\alpha 1$, fibronectin1, N-cadherin, vimentin and S100A4) and EMT-related transcription factors (Snail1 and Slug) in activated PSCs (10 days after isolation). Data are expressed as mean \pm SD from three biological replica, ${ }^{*} \mathrm{P}<0.05$. 


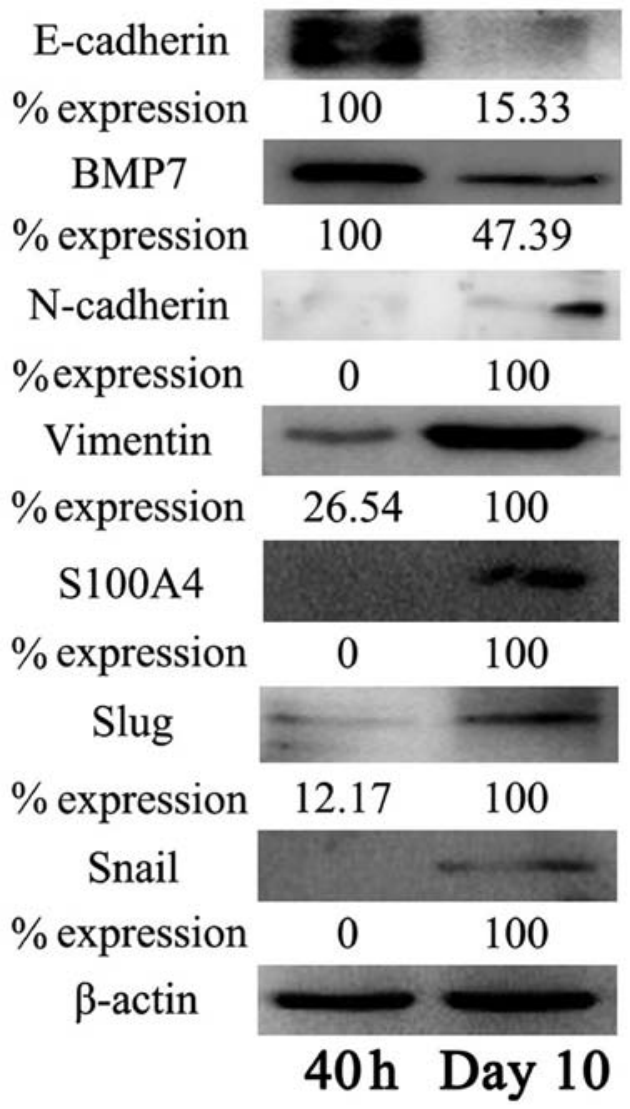

Figure 7. Western blotting indicating alterations in protein expression in activated pancreatic stellate cells (PSCs). In contrast to quiescent PSCs (40 h after isolation), the protein levels of the epithelial markers (E-cadherin and BMP7) were downregulated, while mesenchymal markers (collagen $1 \alpha 1$, fibronectin1, N-cadherin, vimentin and S100A4) and transcription factors (Snaill and Slug) were upregulated in activated PSCs (10 days after isolation).

Based on the results of this study and previous studies, it is clear that PSCs complete an EMT-like process that promotes the development of a migratory, mesenchymal phenotype from a quiescent phenotype. Therefore, the EMT process is a key event in PSC activation. It follows that reversing the EMT process could be a potential therapeutic strategy for chronic pancreatitis and pancreatic cancer. However, little is known about the reverse process of EMT, which is called mesenchymalepithelial transition (MET). The process of MET is well known in kidney formation, and multiple genes are involved, including BMP7 (47-49). Similarly, BMP7 has been reported to act as a positive regulator of MET in mice (35). BMP-7 antagonizes TGF- $\beta 1$-induced fibrotic effects in vitro and reverses fibrosis in various organs such as the kidney, heart and colon (50). Therefore, reducing the expression of BMP7 is likely to restore quiescence in activated PSCs. However, further work is required to clarify the molecular mechanisms underlying the signaling pathways involved in EMT and MET processes.

One pathway known to be involved in EMT is the Hedgehog pathway. This pathway promotes formation of mesenchymal cells and has been shown to participate in HSC transdifferentiation from quiescent HSCs to myofibroblastic HSCs in vitro (51-53). Similarly, in vivo studies have indicated that Hedgehog pathway activation is associated with EMT, myofibroblast proliferation and liver fibrosis while inhibition of this pathway attenuates EMT, myofibroblast accumulation and fibrosis in mouse models $(29,54,55)$. For this reason, genes related to the Hedgehog pathway were examined by qRT-PCR in the present study (data not shown). We found the in the activated PSCs, Gli1, Gli2 (i.e., Hedgehog target genes) and sonic hedgehog (i.e., the Hedgehog ligand) were all upregulated, and HHIP (i.e., a Hedgehog ligand antagonist) was downregulated. However, additional research is needed to confirm whether the Hedgehog signaling pathway modulates the process of PSC transdifferentiation.

In conclusion, the present study supports that the transition of quiescent PSCs to activated myofibroblastic PSCs involves an EMT-like process in vitro. This knowledge improves our understanding of the pathogenesis of pancreatic fibrogenesis, and offers a potential theoretical basis for future research on the treatment of PDAC and chronic pancreatitis.

\section{Acknowledgements}

This study was supported in part by the National Natural Science Foundation of China (81272239, 81170336, 81272382, 81300351), the Natural Science Foundation of Jiangsu Province (BK2012881), the Program for Development of Innovative Research Team in the First Affiliated Hospital of NJMU, the Priority Academic Program Development of Jiangsu Higher Education Institutions (PAPD, JX10231801), and the Translational Research of Early Diagnosis and Comprehensive Treatment in Pancreatic Cancer (The research Special Fund For Public Welfare Industry of Health, 201202007).

\section{References}

1. Siegel R, Ma J, Zou Z and Jemal A: Cancer statistics, 2014. CA Cancer J Clin 64: 9-29, 2014.

2. Pandol S, Gukovskaya A, Edderkaoui M, Dawson D, Eibl G and Lugea A: Epidemiology, risk factors, and the promotion of pancreatic cancer: Role of the stellate cell. J Gastroenterol Hepatol 27 (Suppl 2): 127-134, 2012.

3. Chu GC, Kimmelman AC, Hezel AF and DePinho RA: Stromal biology of pancreatic cancer. J Cell Biochem 101: 887-907, 2007.

4. Apte MV, Park S, Phillips PA, Santucci N, Goldstein D, Kumar RK, Ramm GA, Buchler M, Friess H, McCarroll JA, et al: Desmoplastic reaction in pancreatic cancer: Role of pancreatic stellate cells. Pancreas 29: 179-187, 2004

5. Yamaguchi K: How to define patients at high risk for pancreatic cancer. Pancreatology 11 (Suppl 2): 3-6, 2011.

6. Wang W, Liao Z, Li G, Li ZS, Chen J, Zhan XB, Wang LW, Liu F, $\mathrm{Hu} \mathrm{LH}$, Guo Y, et al: Incidence of pancreatic cancer in Chinese patients with chronic pancreatitis. Pancreatology 11: 16-23, 2011.

7. Kudo Y,Kamisawa T, Anjiki H, Takuma K and Egawa N: Incidence of and risk factors for developing pancreatic cancer in patients with chronic pancreatitis. Hepatogastroenterology 58: 609-611, 2011.

8. Pezzilli R, Vecchiarelli S, Di Marco MC, Serra C, Santini D, Calculli L, Fabbri D, Rojas Mena B and Imbrogno A: Pancreatic ductal adenocarcinoma associated with autoimmune pancreatitis. Case Rep Gastroenterol 5: 378-385, 2011.

9. Liotta LA and Kohn EC: The microenvironment of the tumourhost interface. Nature 411: 375-379, 2001.

10. De Wever O and Mareel M: Role of tissue stroma in cancer cell invasion. J Pathol 200: 429-447, 2003.

11. Guerra C, Schuhmacher AJ, Cañamero M, Grippo PJ, Verdaguer L, Pérez-Gallego L, Dubus P, Sandgren EP and Barbacid M: Chronic pancreatitis is essential for induction of pancreatic ductal adenocarcinoma by K-Ras oncogenes in adult mice. Cancer Cell 11: 291-302, 2007.

12. Erkan M, Adler G, Apte MV, Bachem MG, Buchholz M, Detlefsen S, Esposito I, Friess H, Gress TM, Habisch HJ, et al: StellaTUM: Current consensus and discussion on pancreatic stellate cell research. Gut 61: 172-178, 2012. 
13. Jaster R: Molecular regulation of pancreatic stellate cell function. Mol Cancer 3: 26, 2004.

14. Watari N, Hotta Y and Mabuchi Y: Morphological studies on a vitamin A-storing cell and its complex with macrophage observed in mouse pancreatic tissues following excess vitamin A administration. Okajimas Folia Anat Jpn 58: 837-858, 1982.

15. Apte MV, Haber PS, Applegate TL, Norton ID, McCaughan GW, Korsten MA, Pirola RC and Wilson JS: Periacinar stellate shaped cells in rat pancreas: Identification, isolation, and culture. Gut 43: 128-133, 1998.

16. Bachem MG, Schneider E, Gross H, Weidenbach H, Schmid RM, Menke A, Siech M, Beger H, Grünert A and Adler G: Identification, culture, and characterization of pancreatic stellate cells in rats and humans. Gastroenterology 115: 421-432, 1998.

17. Apte M, Pirola R and Wilson J: The fibrosis of chronic pancreatitis: new insights into the role of pancreatic stellate cells Antioxid Redox Signal 15: 2711-2722, 2011.

18. Apte MV, Pirola RC and Wilson JS: Pancreatic stellate cells: A starring role in normal and diseased pancreas. Front Physiol 3 344, 2012.

19. Apte MV, Wilson JS, Lugea A and Pandol SJ: A starring role for stellate cells in the pancreatic cancer microenvironment. Gastroenterology 144: 1210-1219, 2013.

20. Boyer B, Vallés AM and Edme N: Induction and regulation of epithelial-mesenchymal transitions. Biochem Pharmacol 60: 1091-1099, 2000.

21. Scheel C and Weinberg RA: Phenotypic plasticity and epithelialmesenchymal transitions in cancer and normal stem cells? Int J Cancer 129: 2310-2314, 2011.

22. Kalluri R and Neilson EG: Epithelial-mesenchymal transition and its implications for fibrosis. J Clin Invest 112: 1776-1784, 2003.

23. Neilson EG: Plasticity, nuclear diapause, and a requiem for the terminal differentiation of epithelia. J Am Soc Nephrol 18 : 1995-1998, 2007.

24. Thiery JP and Sleeman JP: Complex networks orchestrate epithelial-mesenchymal transitions. Nat Rev Mol Cell Biol 7: 131-142, 2006.

25. Guarino M: Epithelial-mesenchymal transition and tumour invasion. Int J Biochem Cell Biol 39: 2153-2160, 2007.

26. Moustakas A: Integrins open the way to epithelial-mesenchymal transitions. Cell Cycle 9: 1682, 2010.

27. Zeisberg M and Neilson EG: Biomarkers for epithelial-mesenchymal transitions. J Clin Invest 119: 1429-1437, 2009.

28. Kalluri R and Weinberg RA: The basics of epithelial-mesenchymal transition. J Clin Invest 119: 1420-1428, 2009.

29. Choi SS, Omenetti A, Witek RP, Moylan CA, Syn WK, Jung Y, Yang L, Sudan DL, Sicklick JK, Michelotti GA, et al: Hedgehog pathway activation and epithelial-to-mesenchymal transitions during myofibroblastic transformation of rat hepatic cells in culture and cirrhosis. Am J Physiol Gastrointest Liver Physiol 297: G1093-G1106, 2009.

30. Kikuta K, Masamune A, Watanabe T, Ariga H, Itoh H, Hamada S, Satoh K, Egawa S, Unno M and Shimosegawa T: Pancreatic stellate cells promote epithelial-mesenchymal transition in pancreatic cancer cells. Biochem Biophys Res Commun 403: 380-384, 2010

31. Hogan BL: Bone morphogenetic proteins: Multifunctional regulators of vertebrate development. Genes Dev 10: 1580-1594, 1996.

32. Kang MH, Kim JS, Seo JE, Oh SC and Yoo YA: BMP2 accelerates the motility and invasiveness of gastric cancer cells via activation of the phosphatidylinositol 3-kinase (PI3K)/Akt pathway. Exp Cell Res 316: 24-37, 2010.

33. Klahr S: The bone morphogenetic proteins (BMPs). Their role in renal fibrosis and renal function. J Nephrol 16: 179-185, 2003.

34. Ohta S, Schoenwolf GC and Yamada G: The cessation of gastrulation: BMP signaling and EMT during and at the end of gastrulation. Cell Adhes Migr 4: 440-446, 2010.

35. Zeisberg M, Hanai J, Sugimoto H, Mammoto T, Charytan D, Strutz F and Kalluri R: BMP-7 counteracts TGF-beta1-induced epithelial-to-mesenchymal transition and reverses chronic renal injury. Nat Med 9: 964-968, 2003.
36. Zeisberg M, Shah AA and Kalluri R: Bone morphogenic protein-7 induces mesenchymal to epithelial transition in adult renal fibroblasts and facilitates regeneration of injured kidney. J Biol Chem 280: 8094-8100, 2005.

37. Tarabykina S, Griffiths TR, Tulchinsky E, Mellon JK, Bronstein IB and Kriajevska M: Metastasis-associated protein S100A4: Spotlight on its role in cell migration. Curr Cancer Drug Targets 7: 217-228, 2007.

38. Mazzucchelli L: Protein S100A4: Too long overlooked by pathologists? Am J Pathol 160: 7-13, 2002.

39. Li ZH and Bresnick AR: The S100A4 metastasis factor regulates cellular motility via a direct interaction with myosin-IIA. Cancer Res 66: 5173-5180, 2006.

40. Zeisberg EM, Potenta S, Xie L, Zeisberg M and Kalluri R: Discovery of endothelial to mesenchymal transition as a source for carcinoma-associated fibroblasts. Cancer Res 67: 10123-10128, 2007.

41. Strutz F, Okada H, Lo CW, Danoff T, Carone RL, Tomaszewski JE and Neilson EG: Identification and characterization of a fibroblast marker: FSP1. J Cell Biol 130: 393-405, 1995.

42. Barrallo-Gimeno A and Nieto MA: The Snail genes as inducers of cell movement and survival: Implications in development and cancer. Development 132: 3151-3161, 2005.

43. Nieto MA: The snail superfamily of zinc-finger transcription factors. Nat Rev Mol Cell Biol 3: 155-166, 2002.

44. Cano A, Pérez-Moreno MA, Rodrigo I, Locascio A, Blanco MJ, del Barrio MG, Portillo F and Nieto MA: The transcription factor snail controls epithelial-mesenchymal transitions by repressing E-cadherin expression. Nat Cell Biol 2: 76-83, 2000.

45. Batlle E, Sancho E, Francí C, Domínguez D, Monfar M, Baulida J and García De Herreros A: The transcription factor snail is a repressor of E-cadherin gene expression in epithelial tumour cells. Nat Cell Biol 2: 84-89, 2000

46. Scarpa M, Grillo AR, Brun P, Macchi V, Stefani A, Signori S, Buda A, Fabris P, Giordani MT, De Caro R, et al: Snail1 transcription factor is a critical mediator of hepatic stellate cell activation following hepatic injury. Am J Physiol Gastrointest Liver Physiol 300: G316-G326, 2011.

47. Lipschutz JH: Molecular development of the kidney: A review of the results of gene disruption studies. Am J Kidney Dis 31: 383-397, 1998.

48. Hogan BL and Kolodziej PA: Organogenesis: Molecular mechanisms of tubulogenesis. Nat Rev Genet 3: 513-523, 2002.

49. Rothenpieler UW and Dressler GR: Pax-2 is required for mesenchyme-to-epithelium conversion during kidney development. Development 119: 711-720, 1993.

50. Mizuiri S, Hemmi H, Arita M, Tai R, Hattori Y, Muto A, Suzuki Y, Ohashi Y, Sakai K and Aikawa A: Effluent markers related to epithelial mesenchymal transition with adjusted values for effluent cancer antigen 125 in peritoneal dialysis patients. Int J Nephrol 2011: 261040, 2011.

51. Bailey JM, Singh PK and Hollingsworth MA: Cancer metastasis facilitated by developmental pathways: Sonic hedgehog, Notch, and bone morphogenic proteins. J Cell Biochem 102: 829-839, 2007.

52. Katoh Y and Katoh M: Hedgehog signaling, epithelial-tomesenchymal transition and miRNA (review). Int J Mol Med 22: 271-275, 2008.

53. Choi SS, Syn WK, Karaca GF, Omenetti A, Moylan CA, Witek RP, Agboola KM, Jung Y, Michelotti GA and Diehl AM: Leptin promotes the myofibroblastic phenotype in hepatic stellate cells by activating the hedgehog pathway. J Biol Chem 285: 36551-36560, 2010

54. Omenetti A, Porrello A, Jung Y, Yang L, Popov Y, Choi SS, Witek RP, Alpini G, Venter J, Vandongen HM, et al: Hedgehog signaling regulates epithelial-mesenchymal transition during biliary fibrosis in rodents and humans. J Clin Invest 118: 3331-3342, 2008

55. Syn WK, Jung Y, Omenetti A, Abdelmalek M, Guy CD, Yang L, Wang J, Witek RP, Fearing CM, Pereira TA, et al: Hedgehogmediated epithelial-to-mesenchymal transition and fibrogenic repair in nonalcoholic fatty liver disease. Gastroenterology 137: 1478-1488 e1478, 2009. 